Whether or not the rearrangements of epoxides take place via intermediate glycols is being investigated.

The second session was devoted to anionotropy. Prof. W. G. Young described the reactions of allyl alcohol derivatives with thionyl chloride. Under conditions where intervention of chloride ion is avoided, the reactions proceed with rearrangement via cyclic transition states $\left(S_{N} i^{\prime}\right.$ mechanism), for example :

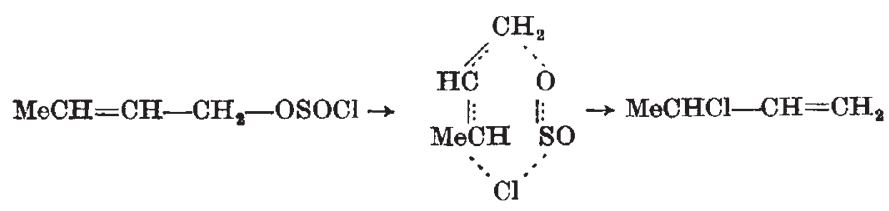

emphasized the difficulty of determining whether or not they are intramolecular. If they are, classical mechanisms will not suffice, for the rearrangements often occur between widely separated centres. $\mathrm{He}$ outlined the $\pi$-complex mechanism for such processes and applied the idea to the rearrangements of $\mathrm{N}$-bromoacetanilide and of hydrazobenzenes. Fresh experimental evidence was cited which suggested that the former reaction is intramolecular; and a general survey of the available evidence was held to support the mechanism in the case of hydrazobenzene derivatives. In the subsequent discussion, his conclusions concerning the mechanism of the N-bromoacetanilide rearrangement were strongly criticized and strongly defended.

It was generally agreed that the sym-

In the presence of chloride ion, an alternative $S_{N} 2$ reaction leads to unrearranged chloride. By suitable choice of the reaction conditions, either unrearranged chloride, or rearranged chloride, can be obtained. An $S_{N}$ l reaction can also be observed in ionizing solvents, the product from either isomeric alcohol then being an identical mixture of the two chlorides.

Dr. E. A. Braude reviewed the historical development of the concept of anionotropy, and emphasized the role of acid catalysis. He then described a reinvestigation of the rearrangement of $\alpha$-phenylallyl $p$-nitrobenzoate into cinnamyl $p$-nitrobenzoate in the presence of isotopically labelled $p$-nitrobenzoic acid in chlorobenzene solution. It was shown that the reaction occurs by two distinct paths, one involving an internal rearrangement (cf. (IV)) and one a bimolecular reaction with the added acid. Both reactions are catalysed by acid. Dr. Braude concluded that no free allylic cations are involved as intermediates, the bimolecular process being of the $S_{N} 2^{\prime}$ type ; and that free allyl cations are probably not involved in anionotropy (see also Nature, May 8, p. 863).

Prof. S. Winstein criticized Dr. Braude's conclusions on the grounds that free ions cannot in any event occur in the solvent used, and that the work described is consistent with the initial formation of an ion-pair, which can either rearrange by 'internal collapse' or react with $p$-nitrobenzoic acid. He thought that free ions might well participate in more polar solvents. A lively discussion followed, on the distinction between ion-pair mechanisms and mechanisms involving cyclic transition-states. The conclusion appeared to be that ion pairs and covalently bound cyclic intermediates are not qualitatively distinct, but form extremes in a graded series (cf. the relation between covalent and ionic bonds). In this case the experimental evidence does not lead to a clear distinction.

The final session dealt with rearrangements in the aromatic series. Prof. E. D. Hughes outlined a general classification of reactions in which groups migrate from the side-chain to the nucleus in an aromatic system. He concluded that the main types of mechanism are an intermolecular process of the $S_{N} 1$ or $S_{N} 2^{\prime}$ type, and an intramolecular process, giving $o$-substituted aniline derivatives via cyclic transition states. The rearrangement of N-phenylhydroxylamine was cited as an example of the former, and the rearrangement of $\mathrm{N}$-nitranilines, and the reaction of aniline with sulphur trioxide, as examples of the latter.

Prof. M. J. S. Dewar outlined the various types of rearrangements that occur in aromatic systems, and

posium had been of value both in presenting various points of view and in clarifying ideas. A remarkable measure of agreement was reached on most of the controversial issues.
M. F. Anselul

M. J. S. DEwar

1 Roberts, J. D., and Lee, C. C., J. Amer. Chem. Soc., 73, 5009 (1951). ${ }^{2}$ Ingold, C. K., and Shoppee, C. W., J. Chem. Soc., 365 (1928).

\section{FRICTION IN RELATION TO RHEOLOGICAL PROPERTIES}

LTHOUGH a number of theories of solid friction A have in the past been proposed, the one which has been most fruitful and most widely accepted in recent years is the adhesion mechanism of friction originally developed for sliding metals. According to this model, metals, when placed in contact, touch only at their high spots, and the local pressures are usually sufficient to produce plastic flow. At these regions strong adhesion occurs by a process which may be called cold welding. The strong junctions thus formed must be sheared if sliding is to occur, and this is primarily the source of the frictional resistance. With metals the deformation of the asperities is primarily plastic. Under these conditions the area of contact is proportional to the load and independent of the size of the bodies, so that the friction, too, is proportional to the load and independent of the size of the bodies (Amontons's laws).

The reality of junction formation is shown by examining the friction tracks, which provide clear evidence of welding and shearing. This raises the interesting question as to why normal adhesion is not observed between metals. Although contaminant films may be partly responsible, the main effect appears to be due to released elastic stresses which peel the surfaces apart as the joining load is removed, so that little or no normal adhesion is detected. With soft ductile materials such as indium, where the metal can take up changes in shape of the surfaces as the load is removed, large adhesions are observed.

\section{Clean Metals and the Influence of Oxide Films}

If metal surfaces are thoroughly denuded of oxide films, Amontons's laws no longer hold. Because the surfaces are clean, they adhere strongly wherever they touch and the sliding process itself produces a very marked growth of junctions. Under favourable conditions, junction growth may proceed until the 
whole of the geometric area is in intimate contact. Gross seizure occurs and ordinary sliding is almost impossible. The presence of small amounts of oxygen, however, enables sliding to occur and the friction is essentially the same as for surfaces prepared in the atmosphere.

The detailed effect of the surface oxide film depends upon the relative rheological properties of the oxide and the metal substrate. If the oxide is hard and the substrate soft, the condition is like a layer of ice on mud. Penetration takes place readily and good metallic contact occurs. The friction is high and the surface damage heavy. If, on the other hand, the oxide is ductile and can flow with the deformed underlying metal, it is better able to maintain its integrity. The friction and surface-damage may be small for moderate deformation; for sufficiently heavy deformations the surface film may be ruptured with a corresponding increase in friction and wear.

The behaviour of aluminium is of particular interest since the oxide is hard and the metallic substrate soft. Consequently, as we should expect, the thin natural oxide film offers little protection to the underlying metal. Some improvement may be obtained by artificially increasing the thickness of the oxide film by anodic oxidation. These films are, in fact, more resistant to rupture than the natural films. At small loads they maintain their integrity and the friction and surface-damage are small. With increasing load the films first fail in tension as a result of the tangential frictional forces produced during sliding. At greater loads gross disintegration of the film occurs. The frictional properties of the films depend on the surface roughness. In addition, their porosity may play an important part in trapping lubricant and so reducing the friction and wear in lubricated sliding.

\section{Brittle Solids}

The two main features of the theory of metallic friction are that cold welding occurs at the interface and that, because plastic flow takes place, the area of contact is proportional to the applied load. With brittle materials it would seem at first sight that neither of these conditions is applicable. A detailed study shows, however, that the local pressures around the individual regions of contact are sufficient to prevent brittle fracture and there is considerable plastic flow at the interface. As a result, although some surface fragmentation may occur, the behaviour is dominated by the plastic deformation of the surface layers. In addition, under the influence of high pressures and plastic flow, strong interfacial adhesion occurs. Consequently, the mechanism derived for metals is applicable; the friction is proportional to the load and of the same order of magnitude as for metals.

The high pressures existing at the points of real contact also play a very important part in boundary lubrication. The lubricant film which is sheared during sliding is subjected to pressures comparable to the yield pressure of the metal (for steel about $200,000 \mathrm{lb} . / \mathrm{sq}$. in.). Under these conditions, the resistance of the film to shear and penetration is enormously increased.

\section{Plastics and Fibres}

Other classes of materials to which the adhesion mechanism of friction may be applied are long-chain polymers, plasties and fibres. The deformation of these materials is not, however, as clearly defined as for metals. At high loads the area of real contact is roughly proportional to the load, so that Amontons's laws are obeyed. At light loads this is no longer so. The coefficient of friction is no longer constant but increases steadily as the load is reduced. Interferometric studies show that the deformation of the surfaces is more nearly elastic than plastic. The area of contact is no longer proportional to the load, and depends on the size and shape of the specimen and on the number of points of contact. Because of this, the friction across fibres may be very different from the friction along them. If the material shows visco-elastic properties, the area of real contact at a given load will increase with the time of loading and there is a corresponding time dependency in the friction.

These and other issues relating elastic, plastic and visco-elastic properties of materials to their frictional behaviour were discussed at a symposium convened by the British Society of Rheology on February 26, on "Friction in relation to Rheological Properties". Dr. L. R. G. Treloar presided and the three main papers were given by Dr. D. Tabor, on the "Effect of Some Physical and Rheological Properties on the Friction and Lubrication of Solids", Dr. H. J. Howell on "Friction and Surface Deformation in Fibres" and Dr. F. H. R. Wright on "Frictional Behaviour of Anodic Films on Aluminium Surfaces".

\section{TABOR}

\section{SCIENCE IN THE UNITED STATES}

7 HE sixth of the annual reviews produced by the British Commonwealth Scientific Office in North America, entitled "Science in the U.S.A. for the Year ended June 1953"*, follows much the same lines as that for the year ended June 1952, which was the first to be openly published (see Nature, 172, $282 ; 1953)$. In its sections on the expenditure of the Federal Government on research and development, on industrial research and development, and on university and non-profit research, it draws extensively on reports issued by the National Science Foundation. A section on scientific and technological man-power is likewise based on two reports, "Student Deferment and National Manpower Policy" and "A Policy for Scientific and Professional Manpower", issued by the National Manpower Council; these are to be followed by a third, "A Policy for Technical and Skilled Manpower". The second of these reports estimates that, of the five millions now engaged in scientific and professional work, more than a million are teachers, nearly a million are engaged in medical and health services and more than half a million are engineers ; but of 155,000 workers in natural science, about 15,000 are engaged in fundamental research. Shortages in different branches of science are regarded as qualitative as much as quantitative, and it is recommended that no attempt should be made to urge school leavers to turn to particular branches of study in conformity with a predetermined national policy. Instead, expansion over the whole field of higher education is suggested, so as to overcome the

- British Commonwealth Scientific Office, North America. The BCSO Review of Science in the U.S.A. for the Year ending June 1953. (United Kingdom Scientific Mission, Canadian Scientiflc Liaison Office, Australian Scientific Liaison Office, New Zealand Scientiflc Liaison Office, South African Scientiflc Liaison Office.) Pp. iv +44 . (London: H.M.S.O., 1954.) 2s. net. 